\title{
A Phase I Study of Cetuximab and Lapatinib In Patients with Advanced Solid Tumor Malignancies
}

\author{
John F. Deeken, MD¹, Hongkun Wang, $\mathrm{PhD}^{2}$, Deepa Subramaniam, $\mathrm{MD}^{2}$, Aiwu Ruth He, \\ MD, PhD ${ }^{2}$, Jimmy Hwang, MD $^{3}$, John L. Marshall, MD², Christina E. Urso, NP2 , Yiru Wang, \\ MS $^{2}$, Corinne Ramos, PhD $^{4}$, Kenneth Steadman, BS ${ }^{2}$, and Michael J. Pishvaian, MD, PhD $^{2}$ \\ ${ }^{1}$ Inova Comprehensive Cancer and Research Institute, Falls Church VA \\ ${ }^{2}$ Lombardi Comprehensive Cancer Center, Georgetown University Medical Center, Washington, \\ DC \\ ${ }^{3}$ Levine Cancer Center, Carolinas HealthCare System, Charlotte, NC \\ ${ }^{4}$ Theranostics Health, Inc., Rockville MD
}

\begin{abstract}
Background-Acquired resistance to anti-EGFR therapy may be due to EGFR-ErbB2 heterodimerization and pathway reactivation. In pre-clinical studies. inhibiting ErbB2 blocked this resistance mechanism and re-sensitized cells to anti-EGFR therapy. Cetuximab targets the EGFR receptor, whereas lapatinib inhibits both EGFR and ErbB2. We conducted a phase I trial to assess the safety, dose-limiting toxicities (DLTS), and maximum-tolerated dose (MTD) of cetuximab and lapatinib in patients with solid tumors.
\end{abstract}

Methods-Patients received standard weekly cetuximab with escalating lapatinib dosages of 750,1000 or $1250 \mathrm{mg}$ daily in 3-week cycles. DLTs were monitored through the end of cycle 2. Pre- and post-treatment tumor biopsies and germ-line DNA were obtained for correlative studies.

Results-Twenty-two patients were enrolled, and 18 each were evaluable for toxicity and response. Fifty-nine percent had prior anti-EGFR therapy. Common toxicities included rash and diarrhea. No patient experienced a DLT at the highest dose level and no grade 4 toxicity was observed. Response included no CRs, 3 PRs, 9 SD, and 6 DP, for an overall response rate of $17 \%$ and a clinical benefit rate of $67 \%$. The clinical benefit rate in patients previously treated with antiEGFR therapy was 70\%. Mean treatment duration was 4.7 cycles (range 1-14). Decreased expression of EGFR/ErbB2 pathway components after treatment correlated with response, while increased expression in PI3K, Jak/Stat, and MAPK pathways occurred in non-responders.

Conclusions-The combination of cetuximab and lapatinib was well tolerated, with expected toxicities and notable clinical activity, including in patients with previous anti-EGFR therapy. Further clinical study is warranted.

Correspondence: John Deeken, M.D. ICCRI, 3300 Gallows Road, Falls Church VA, 22042, USA. john.deeken@inova.org Tel: +1 (703) 776-8161; Fax: +1 (703) 776-8713.

Disclosure: No other authors report a financial conflict of interest.

Interim and final results were presented at the 2012 and 2013 American Society of Clinical Oncology (ASCO) Annual Meetings 


\section{Keywords}

Phase I; EGFR; ErbB2; cetuximab; lapatinib; clinical trial; solid tumors

\section{Introduction}

Successful treatments designed to block epidermal growth factor receptor (EGFR) signaling include monoclonal antibodies targeting the extracellular domain of the EGFR receptor (cetuximab and panitumumab) as well as inhibitors of the intracellular tyrosine kinase domain of the receptor (erlotinib, afatinib, and gefitinib). Cetuximab is a chimeric monoclonal antibody that is FDA-approved for the treatment of metastatic colorectal and head and neck cancers, and targets the extracellular domain of the receptor when it is in its homodimer configuration.

Numerous models explaining acquired resistance to anti-EGFR therapy have been proposed. [1-3] One such proposed mechanism of acquired resistance is via EGFR heterodimerization with ErbB2 (Her2neu), reactivating the EGFR pathway. [3-8] Combining anti-EGFR therapy with anti-ErbB2 targeted agents overcame acquired anti-EGFR resistance in preclinical models of colorectal, head and neck, and lung cancer cell lines. [3, 5-9] For example, gefitinib-resistant non-small cell lung cancer cells had high levels of EGFR and ErbB2 dimerization which in turn led to persistent pathway activation. [9] The addition of lapatinib in this model reduced EGFR and ErbB2 heterodimerization as well as EGFR tyrosine residue phosphorylation, leading to diminished phosphorylation of the PI3K and MAPK pathways. The combination of cetuximab and gefitinib was not synergistic, but the combination of cetuximab and lapatinib was synergistic in decreasing cell surface EGFR expression, reducing EGFR phosphorylation, and inducing apoptosis, all leading to higher rates of cell death. [9]

Based on these pre-clinical studies, we conducted a phase I dose finding clinical study of the combination of cetuximab and lapatinib in patients with diseases for which there was evidence at the time of the study that they could treated efficaciously with cetuximab, including KRAS wildtype colorectal cancer, head and neck, non-small cell lung, and anal cancers. [10-13] As part of the study, we performed correlative tumor biopsy and pharmacogenetic studies to explore whether tumor pathway activation and germline genetic variations correlated with response and toxicity.

\section{Methods}

Patients

Adult patients with refractory solid tumors treatable with cetuximab at the time of the study (Kras-wildtype colorectal, non-small cell lung, head and neck, and anal squamous cell cancers) were enrolled in a single-institution, open-label, dose escalation, sequential cohort phase I clinical study at the Lombardi Comprehensive Cancer Center of Georgetown University. Patients were required to have measurable disease, adequate organ function, an ECOG performance status of 0-2, a normal ventricular ejection fraction, a life expectancy of 
greater than 3 months, and resolution of reversible toxicities related to prior therapy to grade 1 or less.

\section{Trial Design and Objectives}

A $3+3$ dose escalation design was used to determine the maximally tolerated dose (MTD) of the combination of lapatinib and cetuximab. Secondary objectives included determining clinical activity as well as translational studies described below. Cycle length was three weeks, with response assessed every two cycles. Initial dose cohorts included 3 patients. If 1 of 3 patients experienced a dose limiting toxicity (DLT), then an additional 3 patients were enrolled at that dose level. If 2 of 6 patients experience a DLT, then the next lower dose of lapatinib would be determined to be the MTD and recommended phase II dose (RP2D) when used in combination with cetuximab. At least 6 patients were to be treated at the RP2D.

\section{Treatment}

Treatment included standard cetuximab dosing of $400 \mathrm{mg} / \mathrm{m}^{2}$ on day 1 , then $250 \mathrm{mg} / \mathrm{m}^{2}$ weekly thereafter. Premedications included acetaminophen $650 \mathrm{mg}$ orally and diphenhydramine 25 to $50 \mathrm{mg}$ intravenously. Three dose cohorts of daily lapatinib were planned at dosages of $750 \mathrm{mg}$ ( 3 tabs), 1,000mg (4 tabs), and 1,250mg (5 tabs) per day. This was due to the fact that while the highest approved dose of lapatinib is $1,500 \mathrm{mg} / \mathrm{day}$, the highest approved dose when combined with another agent (capecitabine) is $1,250 \mathrm{mg} / \mathrm{day}$. Lapatinib started on day 1, and had to be taken at least one hour before or after a meal.

All patients were treated with a daily rash prevention regimen known to reduce rash severity in patients treated with EGFR-directed monoclonal antibody therapy of topical $1 \%$ hydrocortisone cream, skin moisturizer, sunscreen, and doxycycline 100mg twice daily, all starting Day -1 of cycle 1. [14]

\section{Response and Toxicity}

Disease status was assessed radiographically every 2 cycles, and response was assessed using RECIST (version 1.1). Toxicities were assessed using the NCI-CTCAE (version 3.0). The dermatological side effects from anti-EGFR therapy often peak 4 weeks or more after starting therapy. For this reason, and since we expected rash to potentially be our doselimiting toxicity, we monitored patients for DLTs through the first two cycles of treatment, for a total of six weeks. This is in contrast to the usual phase I clinical trial design. DLTs were defined as grade 4 neutropenia or thrombocytopenia lasting more than 5 days, neutropenic infection, or other grade 4 hematologic toxicity, as well as any non-hematologic grade 3/4 except alopecia and as follows: for grade 3 acneform rash, a dose delay of cetuximab therapy was allowed for up to 2 weeks; if it persisted it was considered a DLT. If grade 3/4 diarrhea persisted despite optimal supportive care it was a DLT. A cetuximab infusion reaction was not considered a DLT; instead the patient was taken off study and replaced.

For those previously treated with anti-EGFR therapies, we compared the time patients were on those prior therapies to the time they were treated on study with cetuximab and lapatinib. 
Further, for patients whose best treatment response was stable disease or better, we compared the progression free survival (PFS) on study to the PFS duration of the treatment patients received just prior to study entry, using a similar approach proposed by Van Hoff et al [15] by calculating the ratio of PFS on study/PFS on prior therapy.

\section{Pharmacokinetic Analysis}

In order to investigate whether doxycycline caused a drug-drug interaction with lapatinib via inhibition of CYP3A4, for the first six patients weekly trough lapatinib pharmacokinetic (PK) levels were measured prior to that day's treatment during cycle 1 while on doxycycline. Doxycycline was held during cycle 2, and additional weekly trough levels were measured, allowing for intrapatient comparison. PK levels were assessed using a previously validated methodology. [16]

\section{Pharmacogenetic Testing}

Germline genetic variations in proteins involved in the pharmacokinetics and pharmacodynamics of lapatinib and/or cetuximab were tested and correlated with response and toxicity. A total of 12 single nucleotide polymorphisms (SNPs) in 8 genes were tested for (CCND1, ERBB2, EGF, EGFR, Fc $\gamma R I I I A, F C \gamma R I I A, C Y P 3 A 5$, and ABCB1). DNA was extracted from peripheral blood mononuclear cells (PBMCs) obtained from whole blood samples. SNP identification primers (Applied Biosystems, Foster City, CA) along with VIC \& FAM probes were used. Samples were run on a 7900HT Real Time PCR System (AME Bioscience, Toroed, Norway) at 40 Cycles (at $95^{\circ} \mathrm{C}$ for 15 seconds followed by $60^{\circ} \mathrm{C}$ for 60 seconds). Data analysis was performed with the SDS v.2.1 software (Applied Biosystems, Foster City, CA).

\section{Tumor Biopsy Analysis}

Patients underwent tumor biopsies within 4 weeks of starting treatment and then at the end of cycle 1 . Two biopsies were obtained at each time point. One was immediately placed on dry ice, and transported to $-80^{\circ} \mathrm{C}$ freezer storage in less than 15 minutes to ensure integrity of protein phosphorylation status. A second was placed in formalin and embedded in paraffin. Protein lysates were prepared from each frozen sample, and robotically immobilized on nitrocellulose-coated slides along with positive and negative controls. Each array was interrogated with a highly specific antibody that targeted a protein -- including specific phosphorylated sites on such proteins -- involved in the EGFR-pathway, using a proprietary reverse phase protein microarray (RPMA) platform. Paraffin embedded samples were analyzed using the same antibodies to these target proteins.

A total of 32 proteins were assessed in the EGFR/ErbB2 and related downstream pathways, including MAPK, PI3 Kinase, Jak/Stat, and p38 MAPK. The involvement of others tyrosine kinase receptors were also studied, including c-Met and Insulin receptors. Intensity values within each independent spot were subjected to local background subtraction and subtraction of values from non-specific binding of the secondary antibody using a matched slide exposed to the secondary antibody alone. Results were checked manually for saturation and limits of detection. Staining intensity was normalized to the total protein intensity value 
within the same spot by staining each slide with Sypro Ruby Red total protein stain prior to primary antibody application.

\section{Statistical Methods}

The exact Cochran-Armitage test was used to determine whether genotype and the count of genetic polymorphisms were associated with either toxicity or efficacy parameters. Tumor expression levels of the EGFR and ErbB2 pathway proteins were analyzed in two ways: 1) Spearman's Rank Correlation Coefficient was used to assess the relationship between the baseline measures determined from paraffin embedded samples and response; and, 2) paired specimens were described as the percent change from baseline to that measured in a biopsy after one cycle of therapy. Any of the exploratory inferences were considered interesting for further exploration in later trials if the p-value was less than 0.10 .

The protocol was approved by the Lombardi Comprehensive Cancer Center's scientific review committee and the Medstar/Georgetown University's cancer-specific institutional review board, and overseen by the cancer center's data and safety monitoring committee.

\section{Results \\ Patients}

Between October, 2010 and October, 2012, a total of 22 patients were enrolled and started on therapy (Table 1). Patients had colorectal $(n=8)$, non-small cell lung $(n=8)$, head and neck $(\mathrm{n}=4)$, and anal $(\mathrm{n}=2)$ cancers. The median age was 62 years, and a majority of patients were female. Patients were heavily pretreated, with a median of 3.6 lines of prior therapy for their metastatic/recurrent disease. A total of 13 patients (59\%) had been previously treated with anti-EGFR therapy.

\section{Treatment and Toxicities by Dose Level}

Seven patients were enrolled on dose level 1 . The first treated patient experienced a grade 3 rash by C1D4, which did not resolve in 2 weeks and thus was deemed a DLT. An additional 5 patients were enrolled at that dose level, but no grade 3 rash or other DLT was observed. One patient was taken off-study due to non-compliance. At dose level 2, nine patients were enrolled. One patient experienced grade 3 diarrhea with grade 3 dehydration during cycle 1 despite optimal medical intervention, and thus was a DLT. No other DLTs occurred. Three patients experienced rapid disease progression during the first two cycles, and thus were not evaluable for toxicity and were replaced. At dose level 3 , six patients were enrolled, and none experienced a DLT. One patient progressed after 2 cycles. The other 5 patients were on treatment for 4, 6, 11, 12, and 14 cycles. For all patients, the mean number of cycles received was 4.7 (14.1 weeks, range $1-14)$.

Other toxicities observed were those typical for anti-EGFR therapy, including rash, diarrhea, and fatigue. No grade 4 toxicity was observed. No hepatotoxicity was experienced by any patient.

Toxicities reported during the first two cycles or at any time during treatment that were possibly, probably, or definitely related to treatment are shown in Table 2. Importantly, four 
patients (3 SD, 1 PR; 1 dose level 2, 3 dose level 3) withdrew consent and decided to come off of the study after being on treatment for 4, 6, 13, and 14 cycles due to prolonged mild or moderate (grade 1/2) rash/or fatigue, even after dose modification.

\section{Response}

Of the 22 enrolled patients, 18 were evaluable for response. As a best response, no patient had a complete response (CR), three patients (17\%) experienced a partial response (PR), nine (50\%) had stable disease (SD), and six had disease progression (DP). The patients with PRs had colorectal $(\mathrm{n}=2)$ and head and neck $(\mathrm{n}=1)$ cancers, and the duration on study for these patients was 4,12 , and 12 cycles (3, 9, and 9 months). The clinical benefit rate (SD $+\mathrm{PR}+\mathrm{CR})$ was $67 \%$. At the highest dose level $(\mathrm{n}=6), 1$ patient had DP, 4 had SD, and 1 patient with head and neck cancer had a PR, for a clinical benefit rate of $83 \%$, indicating a possible dose response relationship.

Of the 10 patients evaluable for response who had been previously treated with anti-EGFR therapy, 7 experienced clinical benefit (1 PR in a patient with colorectal cancer and 6 patients with SD). The patient with colorectal cancer had been on single agent cetuximab for 11 months before progressing, then enrolled in this study. Figure 1 is a waterfall plot highlighting response by disease type and prior EGFR therapy.

For patients treated with prior anti-EGFR agents, there was no obvious correlation between the duration of that prior therapy and the duration of being on cetuximab and lapatinib (Figure 2A). There were improvements in PFS for some patients on study compared to the treatment they received just prior to study entry (Figure 2B). For patients whose best response was stable disease or partial response, the average ratio of PFS on study/PFS on prior therapy was 1.6 (range 0.3 to 4.5 ). The study by Von Hoff et al identified a ratio of 1.3 as being clinically beneficial. [15]

\section{Pharmacokinetics}

Complete sets of trough PK measures for cycles 1 and 2 were available for the first 5 patients. There were no statistically significant differences in trough lapatinib PK levels on and off of doxycycline treatment $(\mathrm{p}=0.81)$, indicating no significant drug-drug interaction.

\section{Pharmacogenetic analysis}

Pharmacogenetic testing results indicated that variations in FcrRIIA correlated with clinical benefit (SD+PR vs PD, $\mathrm{p}=0.045)($ Table 3$)$. Variation in $C C N D 1$ correlated with a higher degree of diarrhea in patients with the variant genotype $(\mathrm{p}=0.013)$ and a trend towards improved clinical benefit $(\mathrm{p}=0.07)$. No variant correlated with the degree of rash toxicity.

\section{Tumor Biopsy Analysis}

A total of 18 patients had baseline biopsies successfully performed, and pre- and posttreatment biopsies were available from 11 patients who were also evaluable for response. A decrease in phosphoprotein expression of specific proteins in the EGFR pathway appeared to coincide with clinical benefit (Supporting Information Table 1). In terms of diseasespecific results, for the 5 patients with non-small lung cancer, decreased phosphoprotein 
expression was observed in the patients who experienced stable disease (patient \#15 and \#20), whereas increased protein expression in specific components of the EGFR/ErbB2 and related pathways occurred in the patients who experienced disease progression. This included the PI3 Kinase pathway (\#5 and \#9), MET receptor (\#9 and \#13), MAPK pathway (\#9 and \#13), Jak/Stat pathway (\#13), and IGF protein (\#13).

\section{Discussion}

In the new era of targeted anticancer agent development and treatment, the same questions that were asked in the era of cytotoxic agent development can and should be asked again. First, can active single agents be combined in order to achieve additive or even synergistic efficacy? Second, can agents with overlapping toxicities be combined safely, and how can these toxicities be best managed? Third, when (and not whether) cancer cells develop acquired resistance to an agent, can that acquired resistance be overcome with the addition of other agents?

Anti-EGFR agents have proven efficacy in a range of epithelial solid tumor malignancies, especially in the metastatic setting, either as single therapies or when combined with cytotoxic agents. Combined anti-EGFR therapy with cetuximab and erlotinib was recently found to be efficacious in patients with refractory colon cancer in the phase II DUX trial [17]. On the other hand, dual anti-EGFR therapy using cetuximab and either erlotinib or gefitinib was not as effective in treating patients with non-small lung cancer at least in the phase I setting, [18-19] even though additive activity had been observed in pre-clinical models. [20-21] Lapatinib, as an EGFR inhibitor, would be expected to have similar efficacy to the DUX trial when combined with cetuximab, at least in patients with colorectal cancer. Our results showing a 17\% partial response and 50\% stable disease rate highlight the potential benefit from dual anti-EGFR therapy. In fact, our results are more promising than either single agent lapatinib or trials that combined lapatinib with cytotoxic agents in these same cancers. [22-26]

As expected, toxicities associated with anti-EGFR therapies were common in our trial. Over 90 percent of patients experienced a rash, and over 60 percent experienced diarrhea, though the majority were only grade 1 . Interestingly, these toxicities were not dose-dependent. We believe the aggressive combined anti-rash treatment we used likely kept that toxicity from being more significant - and more dose limiting - than it otherwise would have been. This toxicity profile was similar to that seen when trastuzumab was combined with lapatinib in the phase I setting, though in that trial the maximum tolerated dose of lapatinib was only 1,000mg/day. [27]

We used the cautious and unconventional approach for a phase I trial of monitoring patients for these DLTs through a total of two (rather than one) 3-week cycles. Alternatively we could have had cycles last 6 weeks to assess this DLT window, but we determined that restaging such refractory patients only every 3 months (or 2 cycles) could be detrimental to their care if the treatment was not providing benefit. In the end, our approach was not necessary since the vast majority of grade $2 / 3$ rash and diarrhea occurred -- or at least began -- in the first three weeks of therapy. However, some of our patients did experience 
mounting fatigue and skin rash after prolonged treatment. In fact, four patients ( $3 \mathrm{SD}, 1 \mathrm{PR}$ ) withdrew consent after being on treatment for 4, 6, 13, and 14 cycles due to these toxicities. In these patients the skin rash evolved from acneform to a more diffuse erythematous, nonblanching rash that was mainly on the face.

These results highlight the complexity and difficulty in assessing MTD and DLTs in phase I trials of targeted therapies [28] and in the design of phase I combination drug studies. [29] These results also highlight the fact that therapies that are effective can become cumulatively taxing, even in the absence of acute toxicities. Consideration of integrating treatment breaks or rest periods could be worthwhile in this scenario.

Our hypothesis in conducting this phase I trial was that combined anti-EGFR and antiErbB2 therapy may be beneficial, especially in treating tumors that developed acquired resistance after exposure to anti-EGFR therapy via EGFR/ErbB2 receptor heterodimerization and pathway re-activation. [3,5-9] Pre-clinical work done since the start of our trial by Yonesaka and colleagues confirmed this mechanism of acquired resistance to cetuximab, and showed how combined treatment with lapatinib reversed this resistance. [30] We could not directly test this hypothesis since antibodies measuring the heterodimer configuration of EGFR/ErbB2 could not be optimized for tumor assessment. However, our exploratory results in terms of treatment efficacy, including the PFS on study/PFS on prior therapy ratio averaging 1.6 for patients with stable disease or better, suggest that this treatment strategy may be of benefit, and warrants further testing in the phase II setting.

The study of pharmacogenetics attempts to explain differences in pharmacologic response and toxicity by germ line and/or somatic genetic variations. [31] For cetuximab, mutations in the Kras protein -- which is downstream from the EGFR receptor -- can nullify the benefit from blocking the receptor, leading to persistent intracellular signaling and cellular growth. Our finding that a germ-line SNP in Fc $\gamma$ RIIA correlated with clinical benefit is provocative, confirms other findings, [32-34] and deserves additional exploration. The observed impact by this polymorphism is likely due to cetuximab and not lapatinib therapy given the drug's mechanism of action, and may indicate different activation of antibody-dependent cellular cytotoxicity (ADCC). [35]

Tissue biopsies showed decreased phosphoprotein expression of EGFR/ErbB2-related pathways in patients who benefited from therapy. Non-responders showed an increase in expression of phosphoproteins in one or more EGFR downstream pathways in response to treatment. This included critical members of the PI3 Kinase, MET, MAP Kinase, and Jak/ Stat pathways, many of which can be targeted with approved agents or drugs currently in development. Again, how best to identify such patients prior to treatment initiation remains the clinical challenge.

In conclusion, the maximum tolerated and recommended phase II dose of this combination is cetuximab $400 / 250 \mathrm{mg} / \mathrm{m}^{2}$ weekly and lapatinib $1250 \mathrm{mg}$ po daily. Toxicities of rash and diarrhea were common and manageable, especially with aggressive anti-rash supportive care. Clinical benefit was seen in this small cohort of heavily pretreated patients, including patients previously treated with anti-EGFR therapies. Additional clinical trials in solid 
tumors known to be driven by the epidermal growth factor pathway that can be treated with cetuximab are warranted and are being planned.

\section{Supplementary Material}

Refer to Web version on PubMed Central for supplementary material.

\section{Acknowledgements}

We thank the patients and their families who participated in this trial.

Research support: Financial and pharmaceutical support was provided by GlaxoSmithKline, Inc. The project was partially supported by Award Number P30CA051008 from the National Cancer Institute. The content is solely the responsibility of the authors and does not necessarily represent the official views of the National Cancer Institute or the National Institutes of Health.

The following author has a financial relationship related to the subject of this article: Corinne Ramos is an employee of Theranostics Health which performed correlative studies reported herein.

\section{REFERENCES}

1. Van Emburgh BO, Sartore-Bianchi A, Di Nicolantonio F, et al. Acquired resistance to EGFRtargeted therapies in colorectal cancer. Mol Oncol. 2014; 8:1084-1094. [PubMed: 24913799]

2. Cambridge DR, Pao W, Sequist LV. Acquired resistance to TKIs in solid tumors: learning from lung cancer. Nat Rev Clin Oncol. 2014; 11:473-481. [PubMed: 24981256]

3. Erjala K, Sundvall M, Junttila TT, et al. Signaling via ErbB2 and ErbB3 associates with resistance and epidermal growth factor receptor (EGFR) amplification with sensitivity to EGFR inhibitor gefitinib in head and neck squamous cell carcinoma cells. Clin Cancer Res. 2006; 12:4103-4111. [PubMed: 16818711]

4. Learn PA, Krishnegowda N, Talamantez J, et al. Compensatory increases in Her-2/neu activation in response to EGFR tyrosine kinase inhibition in colon cancer cell lines. J Surg Res. 2006; 136:227231. [PubMed: 17059835]

5. Rajput A, Koterba AP, Kreisberg JI, et al. A novel mechanism of resistance to epidermal growth factor receptor antagonism in vivo. Cancer Res. 2007; 67:665-673. [PubMed: 17234777]

6. Half E, Sun Y, Sinicrope FA. Anti-EGFR and ErbB-2 antibodies attenuate cyclooxygenase-2 expression and cooperatively inhibit survival of human colon cancer cells. Cancer Lett. 2007; 251:237-246. [PubMed: 17189670]

7. Wheeler DL, Huang S, Kruser TJ, et al. Mechanisms of acquired resistance to cetuximab: role of HER (ErbB) family members. Oncogene. 2008; 27:3944-3956. [PubMed: 18297114]

8. Kondo N, Ishiguro Y, Kimura M, et al. Antitumor effect of gefitinib on head and neck squamous cell carcinoma enhanced by trastuzumab. Oncol Rep. 2008; 20:373-378. [PubMed: 18636200]

9. Kim HP, Han SW, Kim SH, et al. Combined lapatinib and cetuximab enhance cytotoxicity against gefitinib-resistant lung cancer cells. Mol Cancer Ther. 2008; 7:607-615. [PubMed: 18347147]

10. Cunningham D, Humblet Y, Siena S, et al. Cetuximab Monotherapy and Cetuximab plus Irinotecan in Irinotecan-Refractory Metastatic Colorectal Cancer. N Engl J Med. 2004; 351:337345. [PubMed: 15269313]

11. Vermorken JB, Mesia R, Rivera F, et al. Platinum-Based Chemotherapy plus Cetuximab in Head and Neck Cancer. N Engl J Med. 2008; 359:1116-1127. [PubMed: 18784101]

12. Pirker R, Pereira JR, Szczesna A, et al. Cetuximab plus chemotherapy in patients with advanced non-small-cell lung cancer (FLEX): an open-label randomised phase III trial. Lancet. 2009; 373:1525-1531. [PubMed: 19410716]

13. Lukan N, Ströbel P, Willer A, et al. Cetuximab-based treatment of metastatic anal cancer: correlation of response with KRAS mutational status. Oncology. 2009; 77:293-299. [PubMed: 19923868] 
14. Lacourture ME, Lacouture EP, Piperdi B, et al. Skin Toxicity Evaluation Protocol with Panitumumab (STEPP), a Phase II, Open-Label, Randomized Trial Evaluating the Impact of a PreEmptive Skin Treatment Regimen on Skin Toxicities and Quality of Life in Patients with Metastatic Colorectal Cancer. J Clin Oncol. 2010; 28:1351-1357. [PubMed: 20142600]

15. Von Hoff DD, Stephenson JJ, Rosen P, et al. Pilot study using molecular profiling of patients' tumors to find potential targets and select treatments for their refractory cancers. J Clin Oncol. 2010; 28:4877-4883. [PubMed: 20921468]

16. Burris HA, Hurwitz HI, Dees EC, et al. Phase I safety, pharmacokinetics, and clinical activity study of lapatinib (GW572016), a reversible dual inhibitor of epidermal growth factor receptor tyrosine kinases, in heavily pretreated patients with metastatic carcinomas. J Clin Oncol. 2005; 23:5305-5313. [PubMed: 15955900]

17. Weickhardt AJ, Price TJ, Chong G, et al. Dual targeting of the epidermal growth factor receptor using the combination of cetuximab and erlotinib: preclinical evaluation and results of the phase II DUX study in chemotherapy-refractory, advanced colorectal cancer. J Clin Oncol. 2012; 30:15051512. [PubMed: 22412142]

18. Ramalingam S, Forster J, Naret C, et al. Dual inhibition of the epidermal growth factor receptor with cetuximab, an IgG1 monocloncal antibody, and gefitinib, a tyrosine kinase inhibitor, in patients with refractory non-small cell lung cancer (NSCLC): a phase I study. J Thorac Oncol. 2008; 3:258-264. [PubMed: 18317068]

19. Guarino MJ, Schneider CJ, Hosford MA, et al. Dual inhibition of the epidermal growth factor receptor pathway with cetuximab and erlotinib: a phase I study in patients with advanced solid malignancies. Oncologist. 2009; 14:119-124. [PubMed: 19182243]

20. Huang S, Armstrong EA, Benavente S, et al. Dual-agent molecular targeting of the epidermal growth factor receptor (EGFR): combining anti-EGFR antibody with tyrosine kinase inhibitor. Cancer Research. 2004; 64:5355-5362. [PubMed: 15289342]

21. Matar P, Rojo F, Cassia R, et al. Combined epidermal growth factor receptor targeting with the tyrosine kinase inhibitor gefitinib (ZD1839) and the monoclonal antibody cetuximab (IMC-C225): superiority over single agent receptor targeting. Clin Cancer Res. 2004; 10:6487-6501. [PubMed: 15475436]

22. Fields AL, Rinaldi DA, Henderson CA, et al. An open-label multicenter phase II study of oral lapatinib (GW572016) as single agent, second-Line therapy in patients with metastatic colorectal cancer. J Clin Oncol, 2005 ASCO Annual Meeting Proceedings. 23:3583.

23. de Souza JA, Davis DW, Zhang Y, et al. A phase II study of lapatinib in recurrent/metastatic squamous cell carcinoma of the head and neck. Clin Cancer Res. 2012; 18:2336-2343. [PubMed: 22371453]

24. Ross HJ, Blumenschein GR Jr, Aisner J, et al. Randomized phase II multicenter trial of two schedules of lapatinib as first- or second-line monotherapy in patients with advanced or metastatic non-small cell lung cancer. Clin Cancer Res. 2010; 16:1938-1949. [PubMed: 20215545]

25. Frank D, Jumonville A, Loconte NK, et al. A phase II study of capecitabine and lapatinib in advanced refractory colorectal adenocarcinoma: A Wisconsin Oncology Network study. J Gastrointest Oncol. 2012; 3:90-96. [PubMed: 22811876]

26. Dennie TW, Fleming RA, Bowen CJ, et al. A Phase I study of capecitabine, oxaliplatin, and lapatinib in metastatic or advanced solid tumors. Clin Colorectal Cancer. 2011; 10:57-62. [PubMed: 21609937]

27. Storniolo AM, Pegram MD, Overmoyer B, et al. Phase I dose escalation and pharmacokinetic study of lapatinib in combination with trastuzumab in patients with advanced ErbB2-positive breast cancer. J Clin Oncol. 2008; 26:3317-3323. [PubMed: 18490651]

28. Greystoke A, Ranson M. Learning from toxicity patterns in phase I trials during the era of mechanism targeted agents. Ann Oncol. 2012; 23:1934-1936. [PubMed: 22510374]

29. Hamberg P, Verweij J. Phase I drug combination trial design: walking the tightrope. J Clin Oncol. 2009; 27:4441-4443. [PubMed: 19704054]

30. Yonesaka K, Zejnullahu K, Okamoto I, et al. Activation of ERBB2 signaling causes resistance to the EGFR-directed therapeutic antibody cetuximab. Sci Transl Med. 2011; 3:99ra96. 
31. Deeken J, Figg W, Bates SE, et al. Toward individualized treatment: Prediction of anticancer drug disposition and toxicity with pharmacogenetics. Anticancer Drugs. 2007; 18:111-126. [PubMed: 17159598]

32. Bibeau F, Lopez-Crapez E, Di Fiore F, et al. Impact of Fc \{gamma $\}$ RIIa-Fc\{gamma $\}$ RIIIa polymorphisms and KRAS mutations on the clinical outcome of patients with metastatic colorectal cancer treated with cetuximab plus irinotecan. J Clin Oncol. 2009; 27:1122-1129. [PubMed: 19164213]

33. Rodriguez J, Zarate R, Bandres E, et al. Fc gamma receptor polymorphisms as predictive markers of Cetuximab efficacy in epidermal growth factor receptor downstream-mutated metastatic colorectal cancer. Eur J Cancer. 2012; 48:1774-1780. [PubMed: 22305465]

34. Sclafani F, Gonzalez de Castro D, Cunningham D, et al. Fc $\gamma$ RIIa and Fc $\gamma$ RIIIa Polymorphisms and Cetuximab Benefit in the Microscopic Disease. Clin Cancer Res. 2014 Jul 1. [Epub ahead of print].

35. Schneider-Merck T, Lammerts van Bueren JJ, Berger S, et al. Human IgG2 antibodies against epidermal growth factor receptor effectively trigger antibody-dependent cellular cytotoxicity but, in contrast to IgG1, only by cells of myeloid lineage. J Immunol. 2010; 184:512-520. [PubMed: 19949082] 


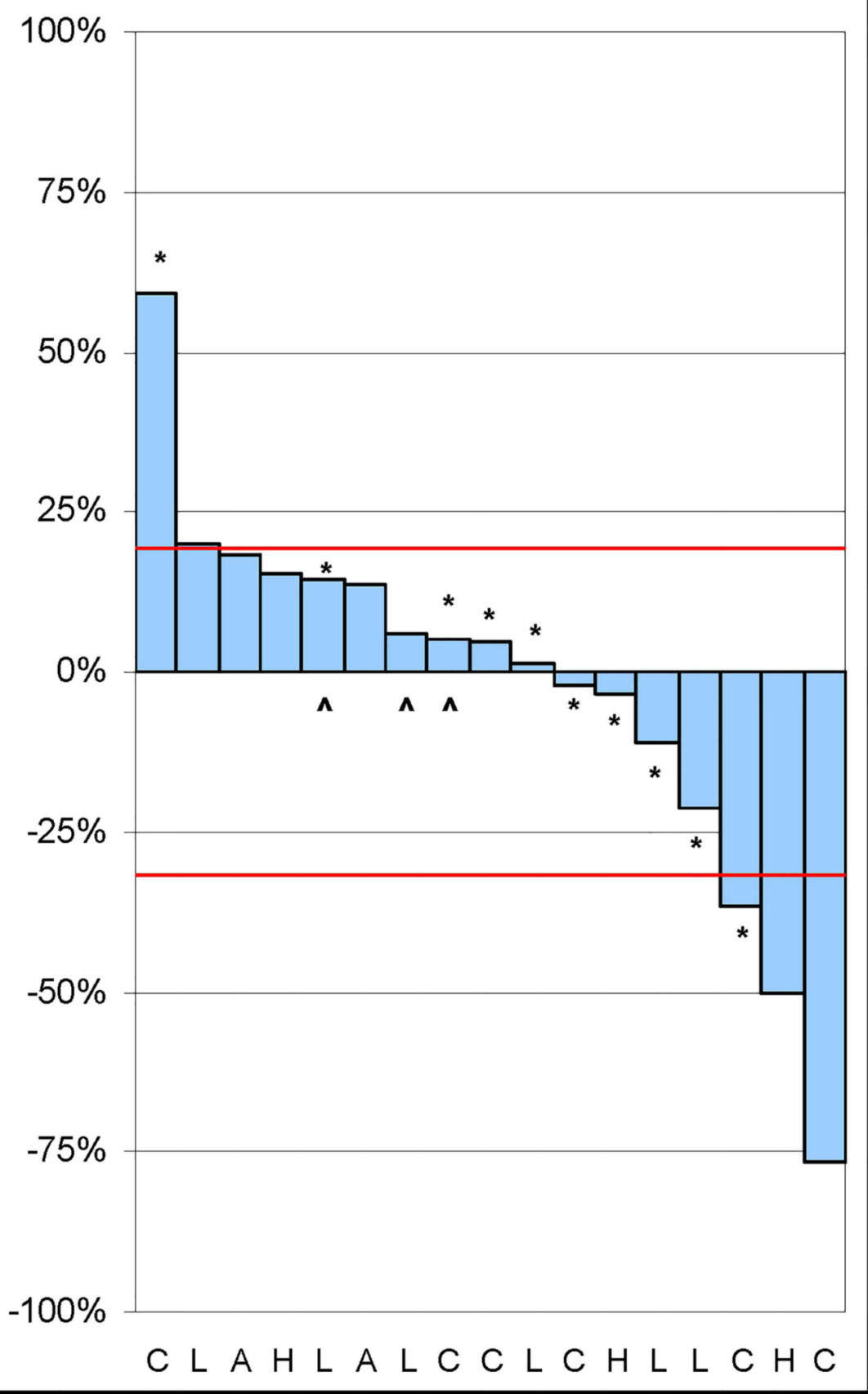

Figure 1.

Waterfall plot of best response by disease type. Patients identified by primary disease as follows: C - colorectal; L - non-small cell lung cancer; A - anal carcinoma; and $\mathrm{H}$ - head and neck cancer. Patients with clinical rather than radiographic progression denoted by ${ }^{\wedge}$. One patient who had tumor progression with new CNS metastases did not undergo complete restaging, and is not included. Patients treated with prior anti-EGFR therapies are indicated by *. 


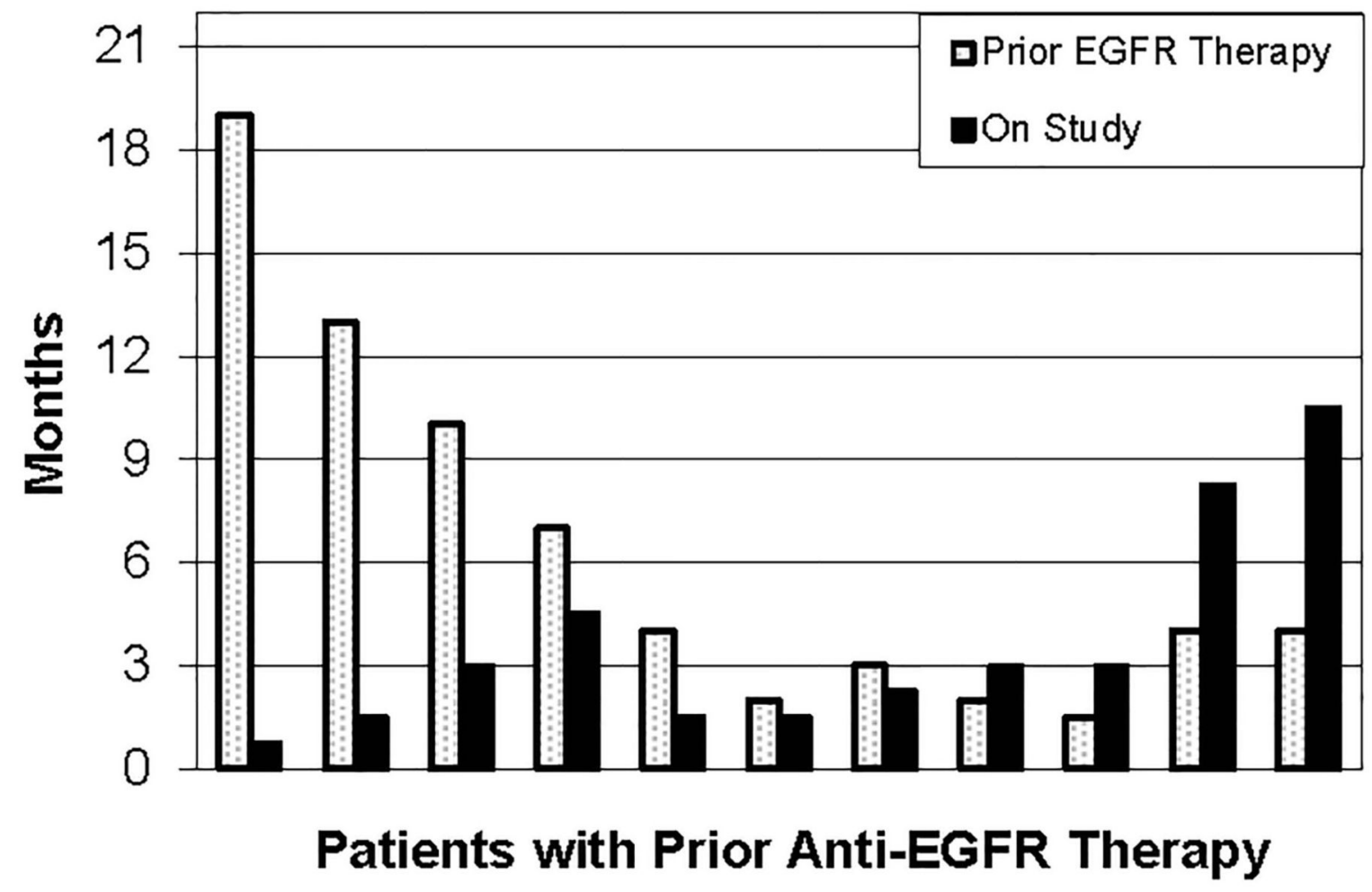

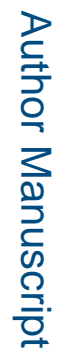

록 


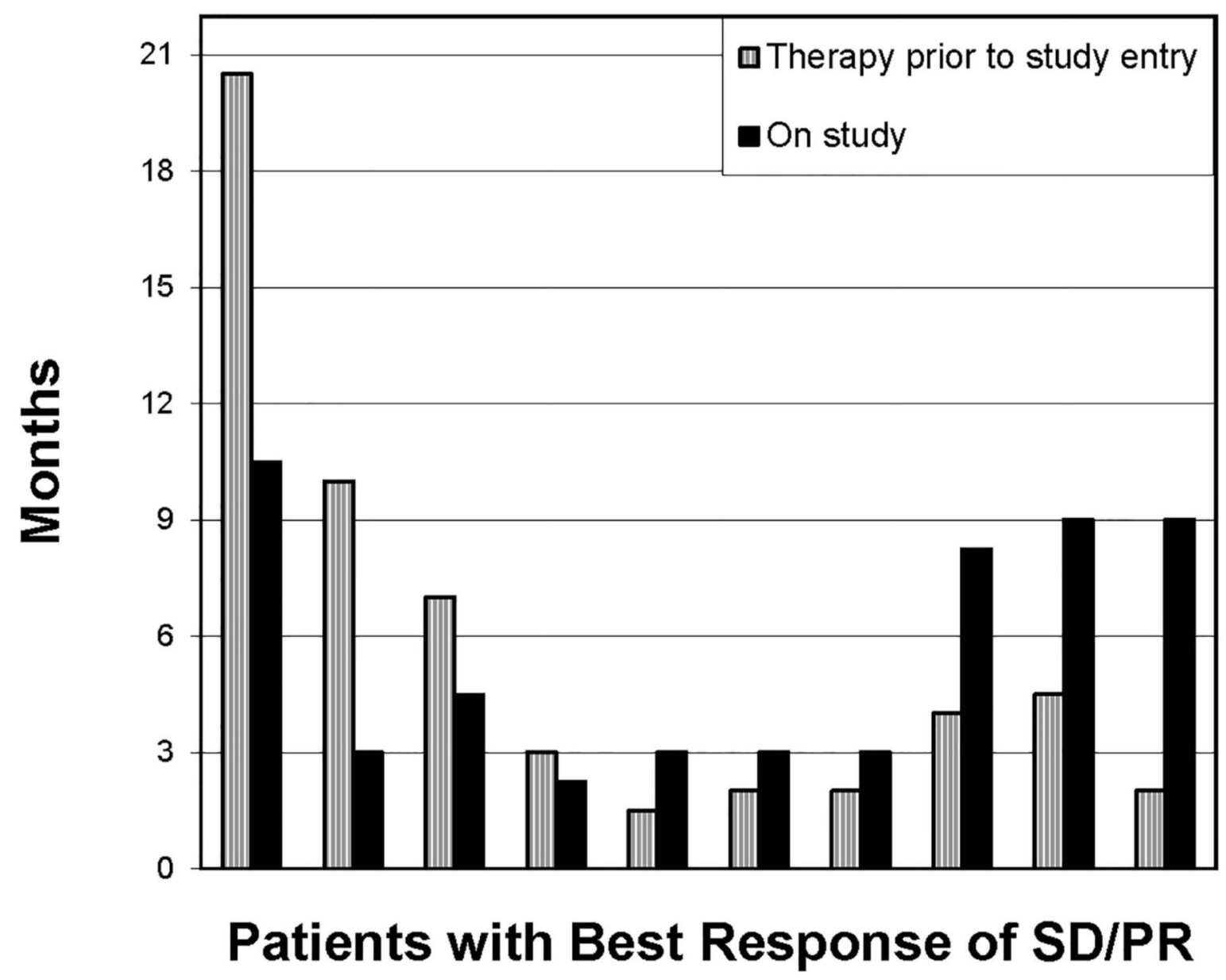

Figure 2.

Duration of treatment by months. Figure A shows duration of prior anti-EGFR therapy compared to duration of treatment on study with cetuximab and lapatinib. Figure B shows duration of prior therapy immediately preceding study entry compared to duration on study with cetuximan and lapatinib for those patients who were treated for more than two cycles and had either stable disease or partial response to treatment. 
Table 1

Demographic information on patients enrolled.

\begin{tabular}{|lc|}
\hline Demographic & Result \\
\hline Total Enrolled & 22 \\
\hline Age - mean (range) years & $62(37-83)$ \\
\hline Gender - male/female & $9 / 13$ \\
\hline Primary Disease & \\
Colorectal & 8 \\
Lung (total) & 8 \\
Adenocarcinoma & 4 \\
Squamous cell & 3 \\
Other & 1 \\
Anal & 2 \\
Head and Neck & 4 \\
\hline Lines of Chemotherapy for Metastatic/Recurrent Disease - mean (range) & $3.6(1-11)$ \\
\hline Prior anti-EGFR therapy & $13(59 \%)$ \\
\hline cetuximab & 8 \\
\hline Erlotinib & 2 \\
\hline Multiple anti-EGFR agents & 3 \\
\hline
\end{tabular}

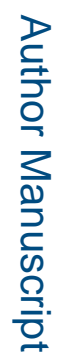


Table 2

Incidence (by number of patients) and grade of treatment-related toxicities experienced during the first two cycles and at any time during treatment.

\begin{tabular}{|l|c|c|c|c|}
\hline \multirow{2}{*}{$\begin{array}{l}\text { Toxicity } \\
\text { experienced by >10\% of patients) }\end{array}$} & \multicolumn{2}{|c|}{ During Cycles $\mathbf{1}$ or 2 } & \multicolumn{2}{c|}{ At Any Time } \\
\cline { 2 - 5 } & $\begin{array}{c}\text { Grade } \\
\mathbf{1 / 2}\end{array}$ & Grade 3 & $\begin{array}{c}\text { Grade } \\
\mathbf{1 / 2}\end{array}$ & Grade 3 \\
\hline DERMATOLOGY/SKIN & & & & \\
\hline Rash & & & & \\
\hline acne/acneiform & 11 & $1^{*}$ & 13 & 2 \\
\hline hand-foot skin reaction & 6 & 0 & 6 & 1 \\
\hline desquamation & 0 & 0 & 2 & 1 \\
\hline dry skin & 0 & 0 & 3 & 0 \\
\hline Pruritus/itching & 0 & 0 & 2 & 1 \\
\hline Mucositis/stomatitis: oral cavity & 3 & 0 & 5 & 0 \\
\hline GASTROINTESTINAL & & & & \\
\hline Diarrhea & 7 & $1 *$ & 10 & 1 \\
\hline Nausea & 6 & 0 & 7 & 0 \\
\hline Vomiting & 3 & 0 & 4 & 0 \\
\hline CONSTITUTIONAL & & & & \\
\hline Fatigue & 5 & 0 & 5 & 1 \\
\hline Anorexia & 3 & 0 & 4 & 0 \\
\hline Pain & 0 & 0 & 2 & 1 \\
\hline HEMATOLOGIC & 0 & 0 & 3 & 0 \\
\hline Hemoglobin & & & & \\
\hline LABORATORY & & & 5 & 1 \\
\hline Magnesium, serum-low & & & 3 & 0 \\
\hline Albumin, serum-low & & & \\
\hline Calcium, serum-low & & & \\
\hline
\end{tabular}

dose limiting toxicities 


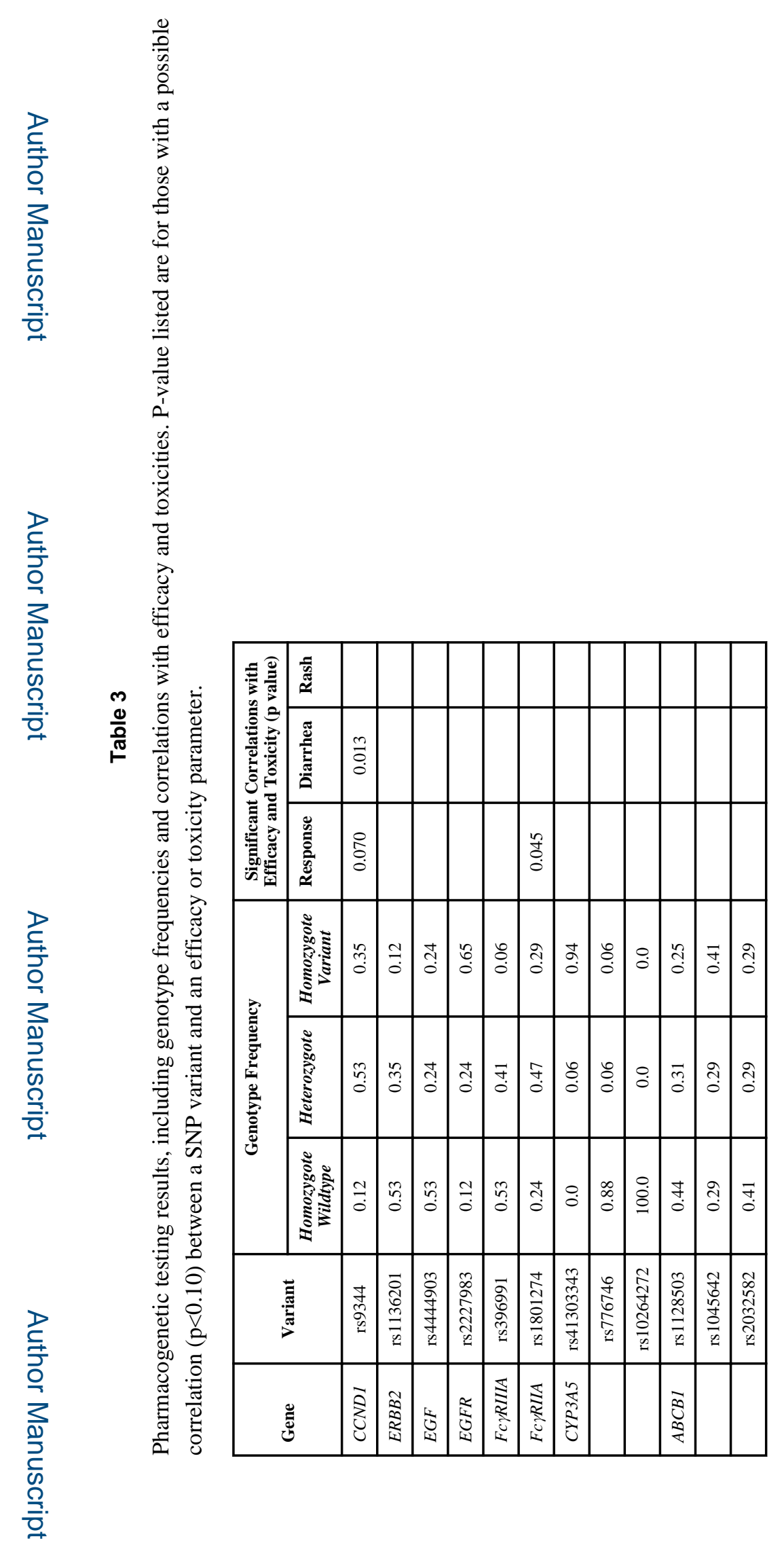

Cancer. Author manuscript; available in PMC 2016 May 15. 